\title{
Polygons with Parallel Opposite Sides
}

\author{
Marcos Craizer • Ralph C. Teixeira • Moacyr A. H. B. da Silva
}

Received: 10 October 2012 / Revised: 15 March 2013 / Accepted: 3 June 2013 /

Published online: 4 July 2013

(C) Springer Science+Business Media New York 2013

\begin{abstract}
In this paper we consider convex planar polygons with parallel opposite sides. These polygons can be regarded as discretizations of closed convex planar curves by taking tangent lines at samples with pairwise parallel tangents. For such polygons, we define discrete versions of the area evolute, central symmetry set, equidistants, and area parallels and show that they behave quite similarly to their smooth counterparts.
\end{abstract}

Keywords Discrete area evolute - Discrete central symmetry set - Discrete area parallels $\cdot$ Discrete equidistants

Mathematics Subject Classification (2010) 53A15

\section{Introduction}

In the emerging field of discrete differential geometry, one tries to define discrete counterparts of concepts from differential geometry that preserve most of the properties of the smooth case. The idea is to discretize not just the equations, but the whole theory [1]. Besides being important in computer applications, good discrete models may shed light

\author{
M. Craizer $(\varangle)$ \\ Departamento de Matemática, PUC-Rio, Rio de Janeiro, Brazil \\ e-mail: craizer@puc-rio.br \\ R. C. Teixeira \\ Departamento de Matemática Aplicada, UFF, Niterói, Brazil \\ e-mail: ralph@mat.uff.br
}

M. A. H. B. da Silva

Centro de Matemática Aplicada, FGV, Rio de Janeiro, Brazil

e-mail: moacyr@fgv.br 
on some aspects of the theory that remain hidden in the smooth context. It is also a common belief that a good discrete counterpart of a differential geometry concept leads to efficient numerical algorithms for computing it.

Several affine symmetry sets and evolutes are associated with closed planar curves. In [3], we considered convex equal area polygons, which can be regarded as discretizations of closed convex planar curves by uniform sampling with respect to affine arc length. For this type of polygon, discrete notions of affine normal and curvature, affine evolute, parallels, and affine distance symmetry set are quite natural.

In this paper we consider convex planar polygons with parallel opposite sides. These polygons can be regarded as discretizations of closed convex planar curves by taking tangent lines at samples with pairwise parallel tangents. For such polygons, we shall define discrete notions of the area evolute, central symmetry set, equidistants, and area parallels and show that they behave quite similarly to the smooth case.

Given a closed convex planar smooth curve, the area evolute is defined as the locus of midpoints of chords with parallel tangents, while the central symmetry set is the envelope of chords with parallel tangents $[4,7]$. It is well known that the area evolute and central symmetry set have the following properties:

- For a point $x$ inside the curve, consider chords with $x$ as midpoint and denote by $N(x)$ the number of such chords. Then $N(x)$ changes by 2 when you traverse the area evolute [4].

- Both the area evolute and the central symmetry set have an odd number of cusps, at least three, and the number of cusps of the central symmetry set is at least the number of cusps of the area evolute [5,10].

- Both the area evolute and the central symmetry set reduce to a point if and only if the curve is symmetric with respect to a point [8].

For a convex polygon with parallel opposite sides, we call great diagonals the lines connecting opposite vertices and define the area evolute and central symmetry set as follows: the central symmetry set is the polygonal line whose vertices are the intersection of great diagonals, while the area evolute is the polygonal line connecting midpoints of the same great diagonals. We also define the notion of cusps for these polygonal lines and then prove that all the aforementioned properties remain true.

An equidistant of a closed convex planar smooth curve is the locus of points that belong to a parallel tangent chord with a fixed ratio with respect to its extremities. The following property is well known:

- The central symmetry set is the locus of cusps of equidistants [4].

We are not aware of any work concerning the set of self-intersections of equidistants.

We define the equidistants of a convex polygon with parallel opposite sides as the polygon whose vertices are along the great diagonals with a fixed ratio with respect to the original vertices. We shall verify that, as in the smooth case, cusps of the equidistants coincide with the central symmetry set. In this discrete setting, the locus of self-intersections of the equidistants is also an interesting set to consider. We call it the equidistant symmetry set and prove that the branches of the equidistant symmetry set have cusps at the central symmetry set and endpoints at cusps of the central symmetry set or cusps of the area evolute. 
For a smooth convex closed planar curve, an area parallel is the envelope of the chords that cut off a fixed area of the interior of the curve [2,9]. The following property is well known:

- The locus of cusps of the area parallels is exactly the area evolute ([6], Sect. 2.7).

For a convex polygon with parallel opposite sides, the definition of area parallels can be carried out without changes, and we obtain piecewise smooth curves made of arcs of hyperbolas. Nevertheless, it seems easier to consider the polygons whose sides connect the endpoints of these arcs of hyperbolas, which we call rectified area parallels. We show that, as in the smooth case, cusps of the rectified area parallels belong to the area evolute.

Given a convex polygon $\mathcal{P}$ with parallel opposite sides, we show the existence of a convex polygon $\mathcal{Q}$ with parallel opposite sides whose central symmetry set is exactly the area evolute of $\mathcal{P}$ and whose sides are parallel to the great diagonals of $\mathcal{P}$.

It is uniquely defined up to equidistants and we call it the parallel-diagonal transform of $\mathcal{P}$.

In the smooth case, the locus of self-intersections of the area parallels is called the affine area symmetry set ([6], Sect. 2.7). For a convex polygon $\mathcal{P}$ with parallel opposite sides, the locus of self-intersections of rectified area parallels will be called the rectified area symmetry set. The rectified area symmetry set is difficult to understand in general, but we can describe it when the original polygon satisfies an almost symmetry hypothesis. A 1-diagonal of $\mathcal{P}$ is a diagonal connecting vertices such that one is adjacent to the opposite of the other. The almost symmetry hypothesis states that for some $\mu_{0}$, the equidistant $\mathcal{Q}_{\mu_{0}}$ of $\mathcal{Q}$ contains the area evolute of $\mathcal{P}$ in its interior and the midpoints of the 1-diagonals are outside it. Under this hypothesis, the equidistants $\mathcal{Q}_{\mu}$ for $\mu \geq \mu_{0}$ coincide with the area parallels of $\mathcal{P}$, and thus the equidistant symmetry set of $\mathcal{Q}$ coincides with the rectified area symmetry set of $\mathcal{P}$. We conclude that the branches of the rectified area symmetry set of $\mathcal{P}$ have cusps at the area evolute of $\mathcal{P}$ and endpoints at the cusps of the area evolute of $\mathcal{P}$ or else at cusps of the area evolute $\mathcal{N}$ of $\mathcal{Q}$. Observe also that, under the almost symmetry hypothesis, $\mathcal{N}$ is exactly the rectified area parallel of level half of the total area of $\mathcal{P}$.

The paper is organized as follows: In Sect. 2 we define the area evolute and central symmetry set for convex polygons with parallel opposite sides and prove their basic properties. In Sect. 3 we study the equidistants and the corresponding symmetry set. In Sect. 4 we define and prove the existence of the parallel-diagonal transform. In Sect. 5 we discuss the properties of the rectified area parallels.

We have used the free software GeoGebra (http://www.geogebra.org/cms) for all figures and many experiments during the preparation of the paper. Applets of some of these experiments are available at http://www.professores.uff.br/ralph (personal home page of the second author), and we refer to them in the text simply as Applets. We would like to thank the GeoGebra team for this excellent mathematical tool.

\section{Basic Notions}

A closed planar polygon $\mathcal{P}$ is called convex if it bounds a convex region and has no parallel adjacent sides. Let $P_{1}, \ldots, P_{n}, P_{n+1}, \ldots, P_{2 n}$ denote the vertices of a convex 


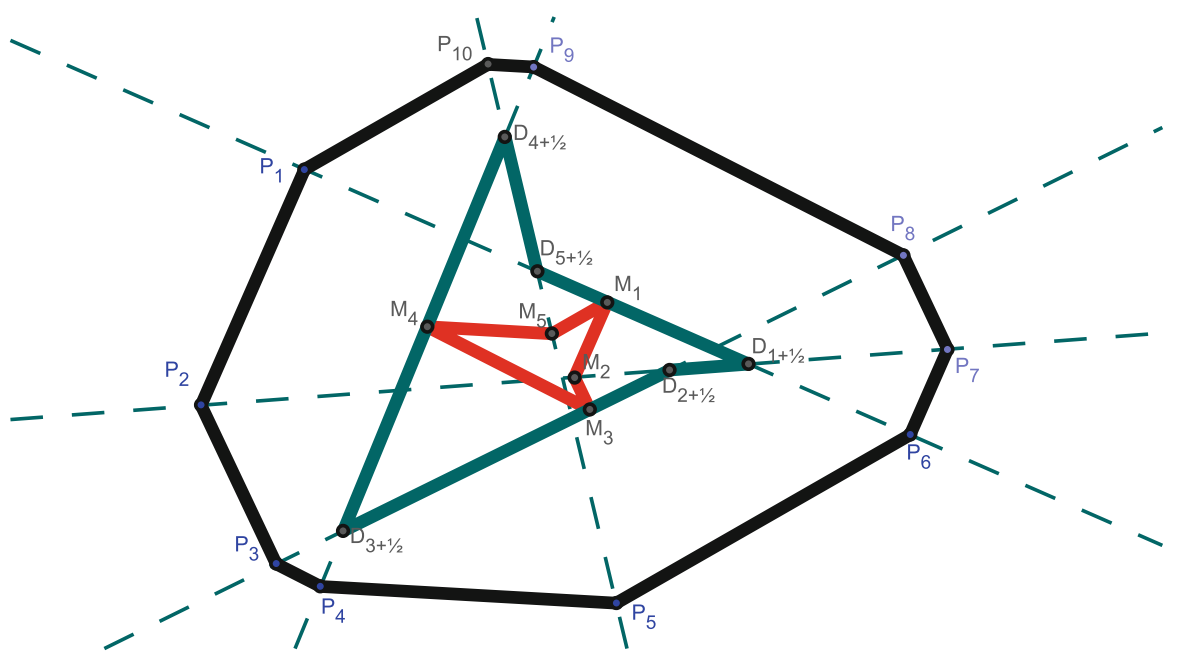

Fig. 1 Great diagonals dashed. The area evolute has vertices $M_{i}$, while the central symmetry set has vertices $D_{i+1 / 2}$

planar $2 n$-gon $\mathcal{P}$. The polygon has parallel opposite sides if

$$
\left(P_{i+n+1}-P_{i+n}\right) \|\left(P_{i+1}-P_{i}\right)
$$

for any $1 \leq i \leq n$. Throughout the paper, indices will be taken modulo $2 n$. We shall assume that the convex parallel opposite sides (CPOS) polygons $\mathcal{P}$ are positively oriented, i.e.,

$$
\left[P_{i+1}-P_{i}, P_{j+1}-P_{j}\right]>0
$$

for any $1 \leq i<j \leq n$.

\subsection{Area Evolute and Central Symmetry Set}

Denote by $d_{i}$ the great diagonal $P_{i} P_{i+n}$ and by $D\left(i+\frac{1}{2}\right)$ the point of intersection of $d_{i}$ and $d_{i+1}$. The central symmetry set of the polygon $\mathcal{P}$ is the polygon whose vertices are $D\left(i+\frac{1}{2}\right), 1 \leq i \leq n$.

Denote by $M_{i}=\frac{1}{2}\left(P_{i}+P_{i+n}\right)$ the midpoints of the segments $P_{i} P_{i+n}$ and by $m\left(i+\frac{1}{2}\right)$ the lines through $M_{i}$ parallel to $P_{i} P_{i+1}$, which we shall call mid-parallel lines. The polygon whose vertices are $M_{i}, 1 \leq i \leq n$, is called the area evolute of the polygon (see Fig. 1 and Applets).

A CPOS polygon is called symmetric with respect to a point $O$ if $P_{i+n}-O=O-P_{i}$ for any $1 \leq i \leq n$.

Proposition 2.1 The central symmetry set of a CPOS polygon reduces to a point if and only if the polygon is symmetric. Similarly, the area evolute of a CPOS polygon reduces to a point if and only if the polygon is symmetric. 
Proof It is clear that if the CPOS polygon is symmetric with respect to a point $O$, then the area evolute and the central symmetry set reduce to this point. It is also clear that if the area evolute of a polygon reduces to a point $O$, then the polygon is symmetric with respect to $O$.

Assume now that the central symmetry set $\mathcal{P}$ reduces to a point $O$. Then necessarily

$$
P_{i+n+1}-P_{i+n}=-\alpha\left(P_{i+1}-P_{i}\right)
$$

for some $\alpha>0$. But such a polygon can close only if $\alpha=1$, and thus $\mathcal{P}$ is symmetric.

\subsection{Mid-Point Property}

We shall denote by $e\left(i+\frac{1}{2}\right)$ the edge whose endpoints are $P_{i}$ and $P_{i+1}$. A pair of edges $e\left(i+\frac{1}{2}\right)$ and $e\left(j+\frac{1}{2}\right)$ determines a parallelogram $M\left(i+\frac{1}{2}, j+\frac{1}{2}\right)$ consisting of the midpoints of pairs $\left(y_{1}, y_{2}\right), y_{1} \in e\left(i+\frac{1}{2}\right)$ and $y_{2} \in e\left(j+\frac{1}{2}\right)$.

Denote by $N(x)$ the number of chords with midpoint $x$, identifying those chords whose endpoints belong to the same sides of the polygon.

Proposition $2.2 N(x)$ is locally constant except at the area evolute. When one traverses a segment of the area evolute, $N(x)$ increases or decreases by 2, depending on the orientation.

Proof Write $x=\frac{1}{2}\left(x_{1}+x_{2}\right)$, where $x_{1} \in e\left(i+\frac{1}{2}\right)$ and $x_{2} \in e\left(j+\frac{1}{2}\right)$. We shall assume that $x_{1}$ and $x_{2}$ are not both endpoints of $e\left(i+\frac{1}{2}\right)$ and $e\left(j+\frac{1}{2}\right)$, or, equivalently, $x$ is not a vertex of $M\left(i+\frac{1}{2}, j+\frac{1}{2}\right)$.

We must consider the following cases:

(1) If $x_{1}$ and $x_{2}$ are both in the interior of $e\left(i+\frac{1}{2}\right)$ and $e\left(j+\frac{1}{2}\right)$, $j \neq i+n$, or, equivalently, $x$ is in the interior of $M\left(i+\frac{1}{2}, j+\frac{1}{2}\right)$, then for $y$ in a neighborhood of $x$, we can find unique $y_{1} \in e\left(i+\frac{1}{2}\right)$ and $y_{2} \in e\left(j+\frac{1}{2}\right)$ such that $y=\frac{1}{2}\left(y_{1}+y_{2}\right)$.

(2) If $x_{1}=P_{i}$ and $j \neq i+n, j \neq i+n-1$, then again there exists a neighborhood $U$ of $x$ such that for $y \in U$ we can find unique $y_{1} \in e\left(i-\frac{1}{2}\right) \cup e\left(i+\frac{1}{2}\right)$ and $y_{2} \in e\left(j+\frac{1}{2}\right)$ such that $y=\frac{1}{2}\left(y_{1}+y_{2}\right)$. In fact, for $y \in U \cap M\left(i-\frac{1}{2}, j+\frac{1}{2}\right)$ we choose $y_{1} \in e\left(i-\frac{1}{2}\right)$, while for $y \in U \cap M\left(i+\frac{1}{2}, j+\frac{1}{2}\right)$ we choose $y_{1} \in e\left(i+\frac{1}{2}\right)$.

(3) Suppose that $x_{1}=P_{i}$ and $x_{2} \in e\left(i+n-\frac{1}{2}\right)$, with $\left|e\left(i-\frac{1}{2}\right)\right|>\left|e\left(i+n-\frac{1}{2}\right)\right|$ (Fig. 2). In this case, $x$ is also the midpoint of $x_{2}^{\prime}=P_{i+n-1}$ and some $x_{1}^{\prime} \in e\left(i-\frac{1}{2}\right)$. Thus, for $y$ in a neighborhood of $x$ we distinguish between $y \in M\left(i+\frac{1}{2}, i+n-\frac{1}{2}\right)$ and $y \in M\left(i-\frac{1}{2}, i+n-\frac{3}{2}\right)$. In the first case, $y$ is the midpoint of $y_{1} \in e\left(i+\frac{1}{2}\right)$ and $y_{2} \in e\left(i+n-\frac{1}{2}\right)$. In the second case, $y$ is the midpoint of $y_{1} \in e\left(i-\frac{1}{2}\right)$ and $y_{2} \in e\left(i+n-\frac{3}{2}\right)$.

(4) It remains to analyze the case where $x$ is on the area evolute. Suppose that $x_{2}=$ $P_{i+n}$ and $x_{1} \in e\left(i-\frac{1}{2}\right)$ with $\left|e\left(i-\frac{1}{2}\right)\right|>\left|e\left(i+n-\frac{1}{2}\right)\right|$ (Fig. 3). In this case, $x$ is 


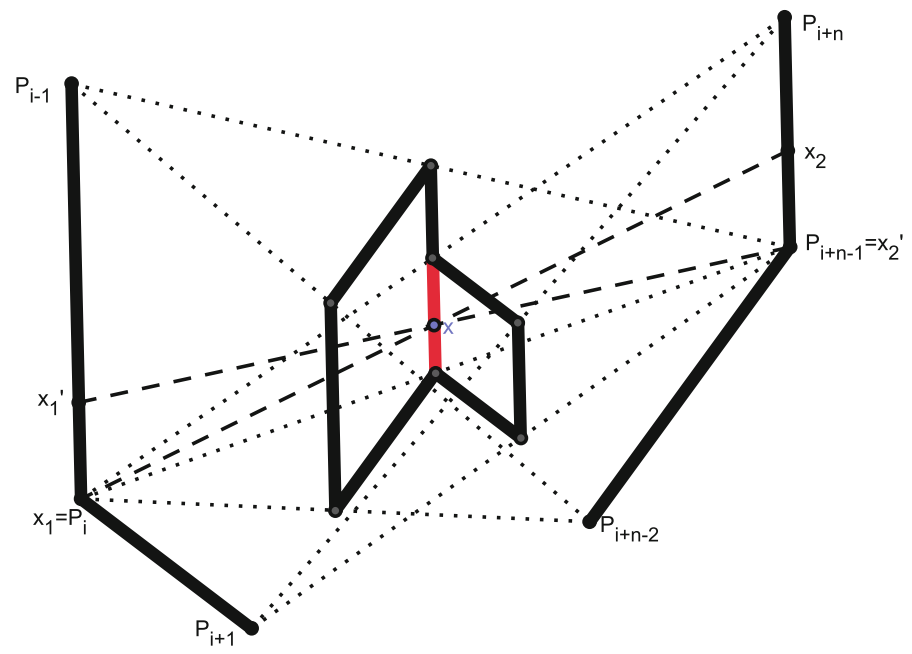

Fig. 2 Case 3. $x$ is in the segment intersection of both parallelograms

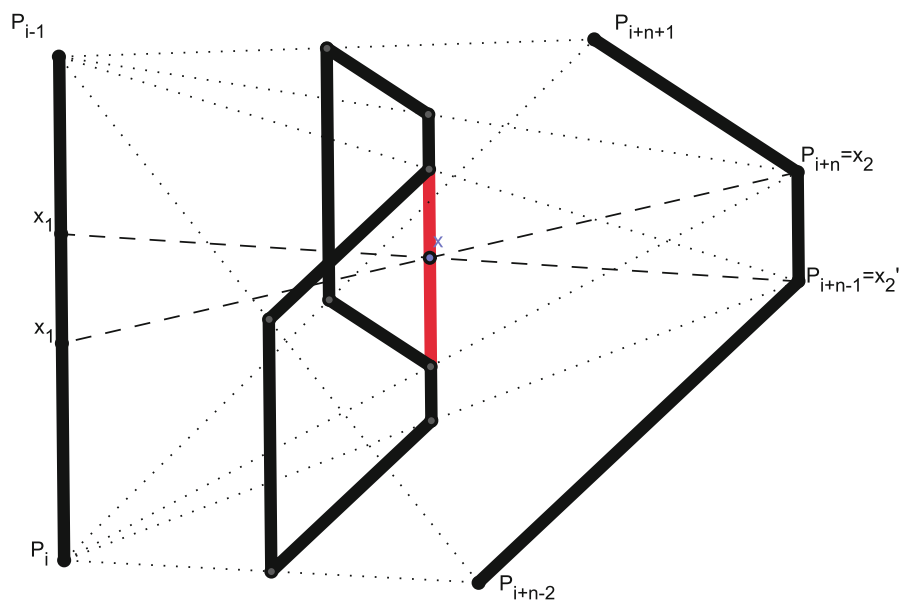

Fig. 3 Case 4. A fold at the area evolute

also the midpoint of $x_{2}^{\prime}=P_{i+n-1}$ and some $x_{1}^{\prime} \in e\left(i-\frac{1}{2}\right)$ and the parallelograms with $x$ in the boundary are $M\left(i-\frac{1}{2}, i+n-\frac{3}{2}\right)$ and $M\left(i-\frac{1}{2}, i+n+\frac{1}{2}\right)$. Consider now $y$ in a neighborhood of $x$. If $y$ is closer to $e\left(i-\frac{1}{2}\right)$, then $y$ is the midpoint of some pair $\left(y_{1}, y_{2}\right), y_{1} \in e\left(i-\frac{1}{2}\right)$ and $y_{2} \in e\left(i+n+\frac{1}{2}\right)$ and is also the midpoint of another pair $\left(y_{1}^{\prime}, y_{2}^{\prime}\right), y_{1}^{\prime} \in e\left(i-\frac{1}{2}\right)$ and $y_{2}^{\prime} \in e\left(i+n-\frac{3}{2}\right)$. On the other hand, if $y$ is closer to $e\left(i+n-\frac{1}{2}\right)$, then there are no pairs $\left(y_{1}, y_{2}\right)$ in the neighborhood of $\left(x_{1}, x_{2}\right)$ or $\left(x_{1}^{\prime}, x_{2}^{\prime}\right)$ with $y$ as midpoint. We conclude that when we traverse a segment of the area evolute from the smaller to the bigger side, $N(x)$ is increased by 2 . 


\subsection{Cusps of Area Evolute and Central Symmetry Set}

In this section, we make the simplifying assumption that $D\left(i-\frac{1}{2}\right) \neq D\left(i+\frac{1}{2}\right)$ for every $1 \leq i \leq n$. Define $\lambda\left(i+\frac{1}{2}\right), 1 \leq i \leq 2 n$, by

$$
D\left(i+\frac{1}{2}\right)=P_{i}+\lambda\left(i+\frac{1}{2}\right)\left(P_{i+n}-P_{i}\right),
$$

where $D\left(i+n+\frac{1}{2}\right)=D\left(i+\frac{1}{2}\right)$. Observe that

$$
e\left(i+n+\frac{1}{2}\right)=\frac{\lambda\left(i+\frac{1}{2}\right)-1}{\lambda\left(i+\frac{1}{2}\right)} e\left(i+\frac{1}{2}\right)
$$

for any $1 \leq i \leq 2 n$ and that $\lambda\left(i+n+\frac{1}{2}\right)=1-\lambda\left(i+\frac{1}{2}\right)$.

We say that a vertex $D\left(i_{0}+\frac{1}{2}\right)$ of the central symmetry set is a cusp if $\lambda\left(i_{0}+\frac{1}{2}\right)$ is a local extremum of the cyclic sequence $\lambda\left(i+\frac{1}{2}\right)$. For the area evolute, we say that a vertex $M_{i_{0}}$ is a cusp if $\lambda\left(i_{0}-\frac{1}{2}\right) \cdot \lambda\left(i_{0}+\frac{1}{2}\right)<0$. The geometrical meaning of these definitions will be clear in the next section.

Proposition 2.3 The number of cusps of the area evolute is odd and $\geq 3$. The number of cusps of the central symmetry set is odd and not smaller than the number of cusps of the area evolute.

Proof Since $\lambda\left(i+n+\frac{1}{2}\right)=1-\lambda\left(i+\frac{1}{2}\right)$, there is an odd number of local extrema and zero crossings in the sequence $\lambda\left(i+\frac{1}{2}\right), 1 \leq i \leq n$. Thus the numbers of cusps of the central symmetry set and the area evolute are odd. Moreover, between two zero crossings there exists at least one local extremum. Thus the number of cusps of the central symmetry set is greater than or equal to the number of cusps of the area evolute.

Assume now by contradiction that the sequence $\lambda\left(i+\frac{1}{2}\right), 1 \leq i \leq 2 n$, has only one zero crossing. Then it would necessarily have $n$ consecutive values greater than $1 / 2$ followed by $n$ consecutive values smaller than $1 / 2$. Thus the sides of the polygon would be of the form $w_{1}, \ldots, w_{n},-\alpha_{1} w_{1}, \ldots,-\alpha_{n} w_{n}$, with $\alpha_{i}>1,1 \leq i \leq n$, and

$$
\sum_{i=1}^{n} w_{i}=\sum_{i=1}^{n} \alpha_{i} w_{i}
$$

Taking the determinant product with $w_{1}$ we get

$$
\sum_{i=2}^{n}\left[w_{1}, w_{i}\right]=\sum_{i=2}^{n} \alpha_{i}\left[w_{1}, w_{i}\right]
$$

which is a contradiction since $\left[w_{1}, w_{i}\right]>0$ for $2 \leq i \leq n$. 
2.4 Symmetric and Nonsymmetric CPOS Equal-Area Polygons

A polygon is called equal-area if

$$
\left[P_{i+1}-P_{i}, P_{i}-P_{i-1}\right]=\left[P_{j+1}-P_{j}, P_{j}-P_{j-1}\right]
$$

for any $i, j$ [3]. It is easy to verify that any symmetric CPOS is equal-area, but, as we shall see below, the reciprocal is true only for even $n$.

For $n$ odd, consider vectors $w_{1}, w_{2}, \ldots, w_{n}$ satisfying $\left[w_{i}, w_{i+1}\right]<0$ and $\sum_{i=1}^{n} w_{i}=0$. For $\alpha>0$, consider a $2 n$-gon $\mathcal{P}$ whose sides are $e_{i}=w_{i}, e_{n+i}=-\alpha w_{i}$ for $1 \leq i \leq n$ odd and $e_{i}=-\alpha w_{i}, e_{n+i}=w_{i}$ for $1 \leq i \leq n$ even. Then the polygon $\mathcal{P}$ is CPOS and equal-area.

Proposition 2.4 Any CPOS equal-area polygon is symmetric or obtained by the foregoing construction.

Proof Let $e_{1}, e_{2} \ldots, e_{n},-\alpha_{1} e_{1}, \ldots,-\alpha_{n} e_{n}$ denote the sides of an equal-area polygon $\mathcal{P}$. Then

$$
\left[e_{1}, e_{2}\right]=\left[e_{2}, e_{3}\right]=\cdots=-\alpha_{1}\left[e_{n}, e_{1}\right]=\alpha_{1} \alpha_{2}\left[e_{1}, e_{2}\right]=\cdots=-\alpha_{n}\left[e_{n}, e_{1}\right] .
$$

Thus $\alpha_{1}=\alpha_{n}$ and $\alpha_{i} \alpha_{i+1}=1$. If $\alpha_{i}=1$ for some $i$, then $\alpha_{i}=1$ for any $i$ and the polygon is symmetric. If $\alpha_{i} \neq 1$, then necessarily $n$ is odd and $\alpha_{i+1}=\alpha_{i}^{-1}$. Thus the polygon is obtained by the foregoing construction.

We shall refer to the preceding constructed polygons as nonsymmetric, equal-area CPOS polygons (see Fig. 4 and Applets).

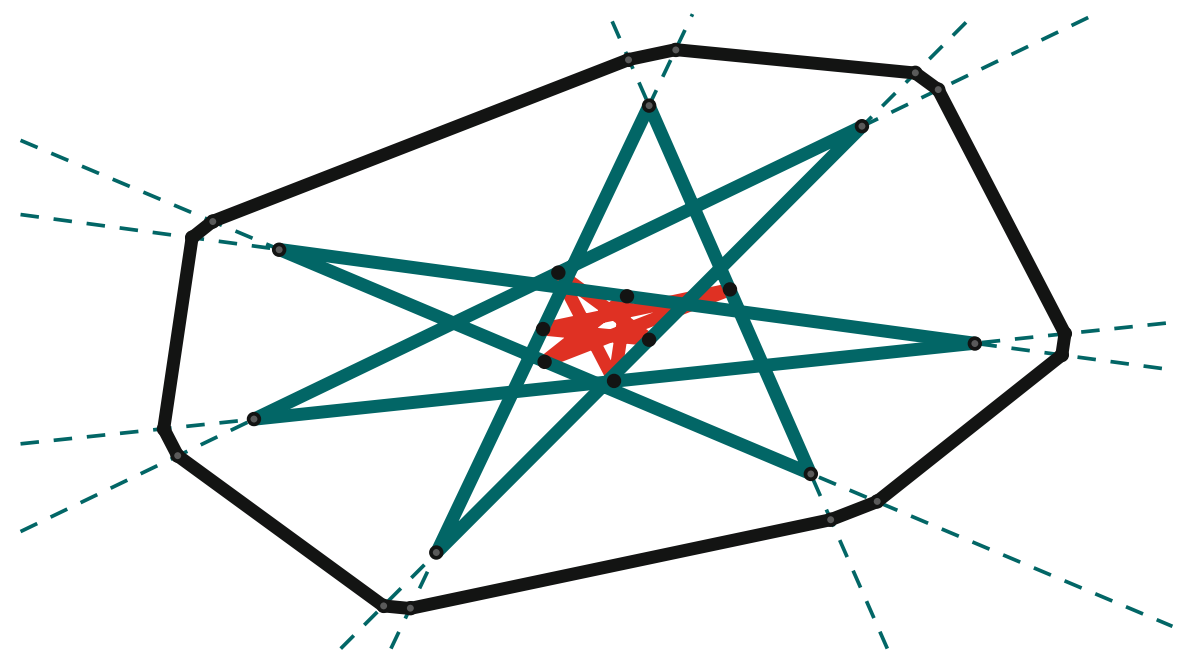

Fig. 4 A CPOS nonsymmetric equal-area 14-gon 


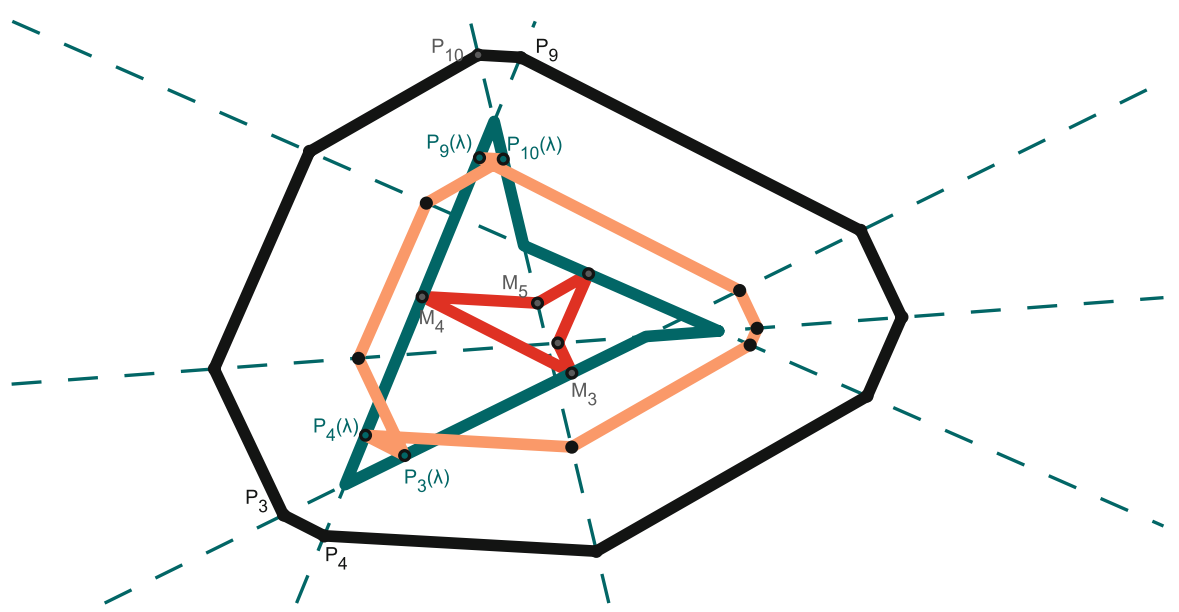

Fig. 5 An equidistant with vertices $P_{i}(\lambda)$ with $\lambda=0.2$. The labeled vertices $P_{i}(\lambda)$ are cusps for this equidistant. When $\lambda=1 / 2$, note that $M_{1}, M_{3}$, and $M_{4}$, which are on the central symmetry set, are cusps, but $M_{2}$ and $M_{5}$ are not

Proposition 2.5 Consider a nonsymmetric, equal-area CPOS polygon $\mathcal{P}$. Then $M_{i}$ is the midpoint of $D\left(i-\frac{1}{2}\right) D\left(i+\frac{1}{2}\right)$. As a consequence, the area evolute and the central symmetry set have $n$ cusps, i.e., all vertices are cusps.

Proof Write $P_{i+1}-P_{i}=-\alpha\left(P_{i+n+1}-P_{i+n}\right)$ for some $\alpha>0$. Then $P_{i+n}-$ $P_{i+n-1}=-\alpha\left(P_{i}-P_{i-1}\right)$. Taking $\lambda=\frac{\alpha-1}{2(1+\alpha)}$, straightforward calculations show that

$$
D\left(i \pm \frac{1}{2}\right)=M_{i} \pm \lambda\left(P_{i+n}-P_{i}\right),
$$

which proves that $M_{i}$ is the midpoint of $D\left(i-\frac{1}{2}\right) D\left(i+\frac{1}{2}\right)$. From this one easily concludes that each point of the central symmetry set is a cusp.

\section{Equidistants}

An equidistant at level $\lambda \in \mathbb{R}$ is a polygon $\mathcal{P}_{\lambda}$ whose vertices are

$$
P_{i}(\lambda)=P_{i}+\lambda\left(P_{n+i}-P_{i}\right)
$$

For $\lambda=\frac{1}{2}$, the equidistant is exactly the area evolute (see Fig. 5 and Applets). The edges of $\mathcal{P}_{\lambda}$ are $e\left(i+\frac{1}{2}\right)(\lambda)=P_{i+1}(\lambda)-P_{i}(\lambda)$. Observe that

$$
e\left(i+\frac{1}{2}\right)(\lambda)=(1-\lambda) e\left(i+\frac{1}{2}\right)+\lambda e\left(i+n+\frac{1}{2}\right)
$$

\subsection{Cusps of the Equidistants and the Central Symmetry Set}

Denote $f_{i}(\lambda)=\left[e\left(i-\frac{1}{2}\right)(\lambda), e\left(i+\frac{1}{2}\right)(\lambda)\right]$. Observe that $f_{i}(0)>0$ and $f_{i}$ changes sign only when $e\left(i-\frac{1}{2}\right)(\lambda)$ or $e\left(i+\frac{1}{2}\right)(\lambda)$ vanishes. Thus $f_{i}(\lambda)<0$ if and only if 


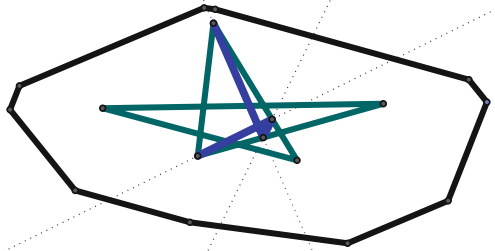

(a) A branch of the equidistant symmetry set with both endpoints at the cusps of the central symmetry set.

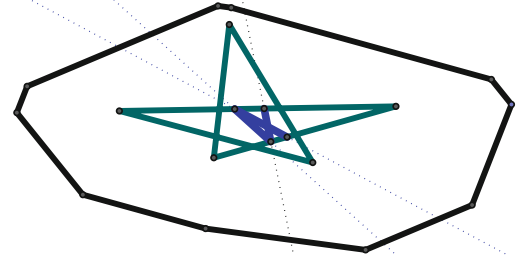

(b) A branch of the equidistant symmetry set with both endpoints at the cusps of the area evolute.

Fig. 6 Branches of equidistant symmetry set

$e\left(i-\frac{1}{2}\right)(\lambda)$ and $e\left(i+\frac{1}{2}\right)(\lambda)$ are in the same half-plane determined by $d_{i}$. We say that a vertex $P_{i}(\lambda)$ is a cusp of the equidistant $\mathcal{P}_{\lambda}$ if $f_{i}(\lambda)<0$.

Proposition 3.1 The set formed by the cusps of the $\lambda$-equidistants coincides with the central symmetry set.

Proof We write

$$
e\left(i \pm \frac{1}{2}\right)(\lambda)=(1-\lambda) e\left(i \pm \frac{1}{2}\right)+\lambda e\left(i+n \pm \frac{1}{2}\right)
$$

Thus $f_{i}(\lambda)$ is a quadratic function that vanishes at $\lambda\left(i-\frac{1}{2}\right)$ and $\lambda\left(i+\frac{1}{2}\right)$ corresponding to the intersections of $d_{i}$ with $d_{i-1}$ and $d_{i+1}$, respectively. Thus $f_{i}(\lambda)<0$ only for $\lambda$ between $\lambda\left(i-\frac{1}{2}\right)$ and $\lambda\left(i+\frac{1}{2}\right)$, which correspond to points of the central symmetry set.

\subsection{Equidistant Symmetry Set}

The self-intersection of the equidistants form a set that we call an equidistant symmetry set (see Fig. 6 and Applets).

Given nonparallel sides $e\left(i+\frac{1}{2}\right), e\left(j+\frac{1}{2}\right)$, denote by $P\left(i+\frac{1}{2}, j+\frac{1}{2}\right)$ the intersection of their support lines. Also, denote by $l\left(i+\frac{1}{2}, j+\frac{1}{2}\right)$ the line passing through $P\left(i+\frac{1}{2}, j+\frac{1}{2}\right)$ and $P\left(i+n+\frac{1}{2}, j+n+\frac{1}{2}\right)$. Observe that the support line of an edge of the equidistant symmetry set associated with a pair of sides $\left(i+\frac{1}{2}, j+\frac{1}{2}\right)$ is exactly $l\left(i+\frac{1}{2}, j+\frac{1}{2}\right)$.

Consider two edges $\left(i-\frac{1}{2}, j+\frac{1}{2}\right)$ and $\left(i+\frac{1}{2}, j+\frac{1}{2}\right)$ of the equidistant symmetry set with a common vertex $\left(i, j+\frac{1}{2}\right)$. We say that the vertex is a cusp if both edges are on the same side of $d_{i}$.

Lemma 3.2 A vertex of the equidistant symmetry set that is not an endpoint is a cusp if and only if it belongs to the central symmetry set.

Proof The support lines of $e\left(i-\frac{1}{2}\right)(\lambda)$ and $e\left(i+\frac{1}{2}\right)(\lambda)$ determine a planar region that does not contain any other support line of $e\left(j+\frac{1}{2}\right)(\lambda), j \neq i-1, i$. We shall denote this region by $R_{i}(\lambda)$. 


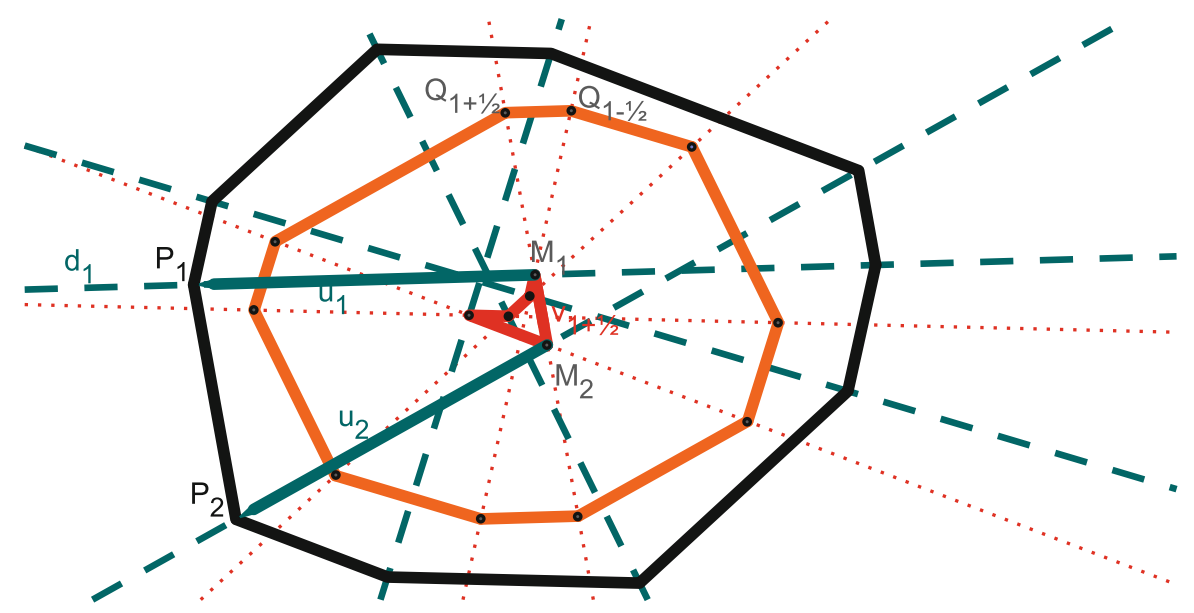

Fig. 7 Parallel-diagonal transform

Consider a vertex $\left(i, j+\frac{1}{2}\right)$ at level $\lambda_{0}$. Assume that $P_{i}\left(\lambda_{0}\right)$ is on the central symmetry set. Then the $\lambda_{0}$-equidistant has a cusp at this point. Observe that the support line of $e\left(j+\frac{1}{2}\right)(\lambda)$ is not contained in $R_{i}(\lambda)$. Thus, for $\lambda$ close to $\lambda_{0}$, the edge $e\left(j+\frac{1}{2}\right)(\lambda)$ intersects $e\left(i+\frac{1}{2}\right)(\lambda)$ and $e\left(i-\frac{1}{2}\right)(\lambda)$ only for $\lambda>\lambda_{0}$ or $\lambda<\lambda_{0}$. For definiteness, we shall assume that these intersections occur for $\lambda>\lambda_{0}$. Thus, for $\lambda>\lambda_{0}$ we have two different intersections of the equidistants; hence the equidistant symmetry set will be on one side of $d_{i}$, which means a cusp.

Conversely, if the vertex $\left(i, j+\frac{1}{2}\right)$ is not on the central symmetry set, then the $\lambda_{0}$-equidistant has a regular point. For $\lambda$ close to $\lambda_{0}$, the edge $e\left(j+\frac{1}{2}\right)(\lambda)$ intersects $e\left(i+\frac{1}{2}\right)(\lambda)$ and $e\left(i-\frac{1}{2}\right)(\lambda)$ once. Thus the corresponding edges of the central symmetry set cross the line $d_{i}$, and thus the vertex is a regular point of the equidistant symmetry set.

Proposition 3.3 Every branch of the equidistant symmetry set can be continued until it reaches a cusp of the central symmetry set or a cusp of the area evolute (Fig. 6).

Proof Consider an edge $e\left(i+\frac{1}{2}, j+\frac{1}{2}\right)$ of the equidistant symmetry set. This edge will end at the great diagonal $d_{i}, d_{i+1}, d_{j}$, or $d_{j+1}$. For definiteness, assume it is $d_{j+1}$. Then we can continue the equidistant symmetry set by the edge $e\left(i+\frac{1}{2}, j+1+\frac{1}{2}\right)$. This is always possible except in two cases: (1) $|j+1-i|=1$ and we are at a cusp of the central symmetry set or (2) $j+1=i+n$ and we are at a cusp of the area evolute.

\section{Parallel-Diagonal Transform}

To a CPOS polygon $\mathcal{P}$ we can associate another CPOS polygon $\mathcal{Q}$ whose vertices are at the mid-parallel lines and whose sides are parallel to the great diagonals of $\mathcal{P}$ (see Fig. 7 and Applets). In this section we prove the existence of such a CPOS polygon $\mathcal{Q}$, called the parallel-diagonal transform of $\mathcal{P}$. 
Denote $v\left(i+\frac{1}{2}\right)=M_{i+1}-M_{i}$ and $u_{i}=P_{i}-M_{i}, 1 \leq i \leq n$. Define also $v\left(i+n+\frac{1}{2}\right)=v\left(i+\frac{1}{2}\right)$ and $u_{i+n}=-u_{i}$. Observe that

$$
\left[v\left(i+\frac{1}{2}\right), u_{i}-u_{i+1}\right]=0
$$

and

$$
\sum_{k=1}^{n} v\left(k+\frac{1}{2}\right)=0
$$

Lemma 4.1 Denote by $A^{1}$ and $A^{2}$ the areas of the regions of the polygon $\mathcal{P}$ bounded by the great diagonal $d_{1}$. Then

$$
A^{2}-A^{1}=2 \sum_{j=1}^{n}\left[v\left(j+\frac{1}{2}\right), u_{j}\right]
$$

Proof Observe that

$$
\begin{aligned}
2 A\left(M_{1} P_{j} P_{j+1}\right) & =\left[\sum_{k=1}^{j-1} v\left(k+\frac{1}{2}\right)+u_{j}, \sum_{k=1}^{j} v\left(k+\frac{1}{2}\right)+u_{j+1}\right], \\
2 A\left(M_{1} P_{j+n} P_{j+n+1}\right) & =\left[\sum_{k=1}^{j-1} v\left(k+\frac{1}{2}\right)-u_{j}, \sum_{k=1}^{j} v\left(k+\frac{1}{2}\right)-u_{j+1}\right],
\end{aligned}
$$

and thus the difference between these areas is

$$
\Delta A(j)=\left[\sum_{k=1}^{j} v\left(k+\frac{1}{2}\right), u_{j}\right]-\left[\sum_{k=1}^{j-1} v\left(k+\frac{1}{2}\right), u_{j+1}\right] .
$$

So $A^{2}-A^{1}$ is given by

$$
\begin{aligned}
\sum_{j=1}^{n} \Delta A(j) & =\sum_{k=1}^{n-1}\left[v\left(k+\frac{1}{2}\right), \sum_{j=k+1}^{n} u_{j}-u_{j+1}\right]+\sum_{j=1}^{n}\left[v\left(j+\frac{1}{2}\right), u_{j}\right] \\
& =\sum_{k=1}^{n-1}\left[v\left(k+\frac{1}{2}\right), u_{k+1}+u_{1}\right]+\sum_{j=1}^{n}\left[v\left(j+\frac{1}{2}\right), u_{j}\right]=2 \sum_{j=1}^{n}\left[v\left(j+\frac{1}{2}\right), u_{j}\right]
\end{aligned}
$$

where we used (4.1) and (4.2).

Denote by $N\left(i+\frac{1}{2}\right)$ the point of the mid-parallel $m\left(i+\frac{1}{2}\right)$ satisfying

$$
\left[N\left(i+\frac{1}{2}\right)-M_{i}, P_{i+n}-P_{i}\right]=\frac{1}{2}\left(A_{i}^{2}-A_{i}^{1}\right),
$$


where $A_{i}^{1}$ and $A_{i}^{2}$ denote the areas of the regions of the polygon $\mathcal{P}$ bounded by the great diagonal $d_{i}$. Denote by $\mathcal{N}$ a polygon with vertices $N\left(i+\frac{1}{2}\right)$.

Proposition 4.2 To a CPOS polygon $\mathcal{P}$, we can associate a CPOS polygon $\mathcal{Q}$ with vertices at the mid-parallel lines and sides parallel to the great diagonals of $\mathcal{P}$. The area evolute of $\mathcal{Q}$ is exactly $\mathcal{N}$.

Proof Start with a point

$$
Q\left(1-\frac{1}{2}\right)=M_{n}+\mu v\left(1-\frac{1}{2}\right) \in m\left(1-\frac{1}{2}\right)
$$

and then follow the parallel to the great diagonal $d_{1}$ until it reaches the line $m\left(1+\frac{1}{2}\right)$ at $Q\left(1+\frac{1}{2}\right)$. Then take the parallel to $d_{2}$ until it intersects the line $m\left(2+\frac{1}{2}\right)$ at $Q\left(2+\frac{1}{2}\right)$. Follow this algorithm and stop after $2 n$ steps, when the polygon reaches the line $m\left(1-\frac{1}{2}\right)$ at $Q\left(2 n+\frac{1}{2}\right)$. We must prove that $Q\left(2 n+\frac{1}{2}\right)=Q\left(1-\frac{1}{2}\right)$.

Define $\mu\left(i+\frac{1}{2}\right), 1 \leq i \leq 2 n$, by the relation

$$
Q\left(i+\frac{1}{2}\right)=M_{i}+\mu\left(i+\frac{1}{2}\right) v\left(i+\frac{1}{2}\right) .
$$

We must then prove that $\mu\left(2 n+\frac{1}{2}\right)=\mu\left(1-\frac{1}{2}\right)$, where $\mu\left(1-\frac{1}{2}\right)=\mu$. Denoting $q_{i}=Q\left(i+\frac{1}{2}\right)-Q\left(i-\frac{1}{2}\right), 1 \leq i \leq 2 n$, we have

$$
q_{i}=\left(1-\mu\left(i-\frac{1}{2}\right)\right) v\left(i-\frac{1}{2}\right)+\mu\left(i+\frac{1}{2}\right) v\left(i+\frac{1}{2}\right)
$$

Since $q_{i}$ must be parallel to $u_{i}$, we obtain

$$
\mu\left(i+\frac{1}{2}\right)\left[v\left(i+\frac{1}{2}\right), u_{i}\right]+\left(1-\mu\left(i-\frac{1}{2}\right)\right)\left[v\left(i-\frac{1}{2}\right), u_{i}\right]=0 .
$$

Denoting $\beta\left(i+\frac{1}{2}\right)=\mu\left(i+\frac{1}{2}\right)-\mu\left(i+n+\frac{1}{2}\right)$, we obtain

$$
\beta\left(i+\frac{1}{2}\right)\left[v\left(i+\frac{1}{2}\right), u_{i}\right]=\beta\left(i-\frac{1}{2}\right)\left[v\left(i-\frac{1}{2}\right), u_{i-1}\right]
$$

where we used (4.1). We conclude that $\beta\left(1-\frac{1}{2}\right)=-\beta\left(n+\frac{1}{2}\right)$, proving that $\mu\left(2 n+\frac{1}{2}\right)=\mu\left(1-\frac{1}{2}\right)$.

Now let

$$
\tilde{N}\left(i+\frac{1}{2}\right)=\frac{1}{2}\left(Q\left(i+\frac{1}{2}\right)+Q\left(i+n+\frac{1}{2}\right)\right)=M_{i}+\frac{1}{2} \gamma\left(i+\frac{1}{2}\right) v\left(i+\frac{1}{2}\right),
$$

where $\gamma\left(i+\frac{1}{2}\right)=\mu\left(i+\frac{1}{2}\right)+\mu\left(i+n+\frac{1}{2}\right)$ denotes the vertices of the area evolute of $\mathcal{Q}$. We have

$$
\left[\tilde{N}\left(i+\frac{1}{2}\right)-M_{i}, P_{i+n}-P_{i}\right]=\gamma\left(i+\frac{1}{2}\right)\left[v\left(i+\frac{1}{2}\right), u_{i}\right]
$$


We claim that $\tilde{N}\left(i+\frac{1}{2}\right)=N\left(i+\frac{1}{2}\right)$ for any $0 \leq i \leq n-1$. The proof of this claim will now be given for $i=0$, the other cases being similar. It follows from (4.3) that

$$
\gamma\left(i+\frac{1}{2}\right)\left[v\left(i+\frac{1}{2}\right), u_{i}\right]=\gamma\left(i-\frac{1}{2}\right)\left[v\left(i-\frac{1}{2}\right), u_{i}\right]+2\left[v\left(i-\frac{1}{2}\right), u_{i}\right] .
$$

Summing from $i=1$ to $i=n$ we obtain

$$
\gamma\left(\frac{1}{2}\right)\left[v\left(\frac{1}{2}\right), u_{1}\right]=\sum_{j=1}^{n}\left[v\left(j+\frac{1}{2}\right), u_{j}\right]=\frac{1}{2}\left(A^{2}-A^{1}\right),
$$

where the last equality follows from Lemma 4.1. Thus the claim is proved.

It remains to show that we can choose $\mu$ such that $\mathcal{Q}$ is convex. Take $\mu$ sufficiently large such that the corresponding $\mathcal{Q}$ contains the area evolute of $\mathcal{P}$ in its interior. For such $\mu$ one easily verifies that $\mathcal{Q}$ has no self-intersections. Since its sides are parallel to $u_{i}, 1 \leq i \leq n$, which are cyclically ordered, we conclude that $\mathcal{Q}$ is convex.

Corollary 4.3 Suppose that the line $P_{i} N\left(i+\frac{1}{2}\right)$ intersects the line $P_{n+i} P_{n+i+1}$ at a point $P_{i}^{\prime}$ at the segment $P_{n+i} P_{n+i+1}$. Then $N\left(i+\frac{1}{2}\right)$ is the midpoint of the chord $P_{i} P_{i}^{\prime}$, which divides the polygon $\mathcal{P}$ into two regions of equal areas.

\section{Rectified Area Parallels}

Consider a simple closed curve $\gamma$ that bounds a convex region. For $x$ inside $\gamma$, there may exist more than one chord $c$ with $x$ as midpoint. Each chord $c$ divides the interior of $\gamma$ into two regions, and we shall denote by $A(x, c)$ the smallest area among these regions. For any $0 \leq \lambda \leq \frac{A(\gamma)}{2}$, the area parallel of level $\lambda$ is the set of points $x$ for which there exists $c$ with $x$ as midpoint and $A(x, c)=\lambda$. Equivalently, the area parallel of level $\lambda$ is the envelope of chords that cut off an area $\lambda$ from the interior of $\gamma$.

\subsection{Area Parallels of a CPOS Polygon}

For each pair of edges $e\left(i+\frac{1}{2}\right)$ and $e\left(j+\frac{1}{2}\right)$, denote by $M\left(i+\frac{1}{2}, j+\frac{1}{2}\right)$ the parallelogram consisting of the midpoints of the edges, as in Sect. 2.2. The area parallels of the wedge bounded by the support lines of $e\left(i+\frac{1}{2}\right)$ and $e\left(j+\frac{1}{2}\right)$ are hyperbolas, and by intersecting any of these hyperbolas with $M\left(i+\frac{1}{2}, j+\frac{1}{2}\right)$ we obtain an arc of hyperbola $H\left(i+\frac{1}{2}, j+\frac{1}{2}, \lambda\right)$ that is part of the area parallel of level $\lambda$ of $\mathcal{P}$.

An area parallel $H\left(i+\frac{1}{2}, j+\frac{1}{2}, \lambda\right)$ necessarily intersects the boundary of the parallelogram $M\left(i+\frac{1}{2}, j+\frac{1}{2}\right)$ at two points. The following lemma describes the line segments $L(\lambda)=L\left(i+\frac{1}{2}, j+\frac{1}{2}, \lambda\right)$ connecting these two points.

Let $A, B, C, D$, and $E$ denote the midpoints of $P_{i+1} P_{j}, P_{i} P_{j}, P_{i+1} P_{j+1}, P_{i} P_{j+1}$, and $P_{i} P_{i+1}$, respectively. Assuming that the area parallel through $B$ intersects the segment $A C$, denote by $F$ this intersection. Then the area parallel through $C$ intersects $B D$ at a point $G$ (Fig. 8). 


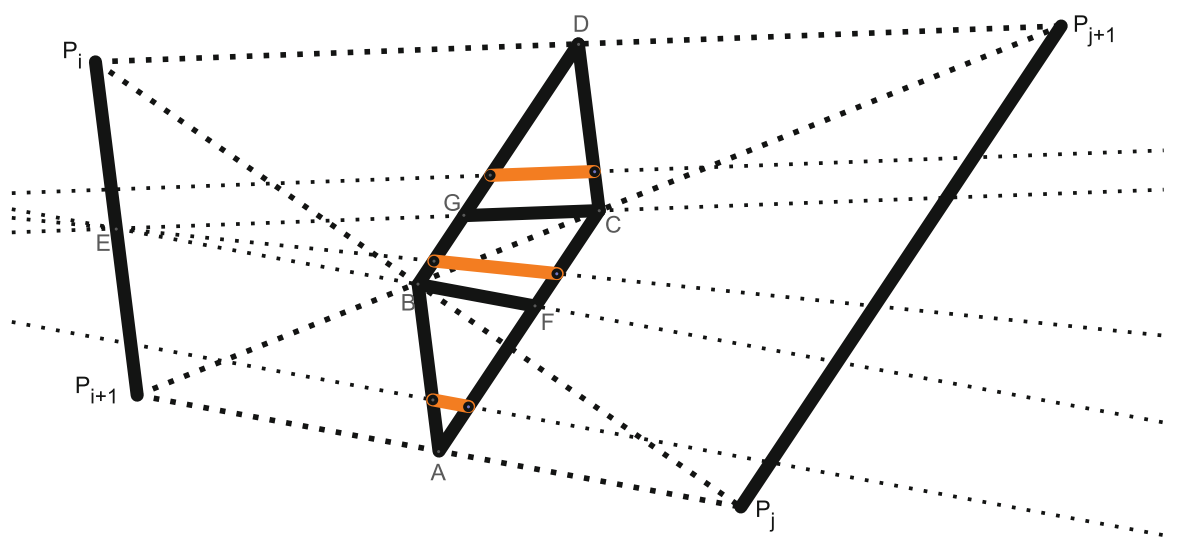

Fig. 8 Area parallels inside a parallelogram

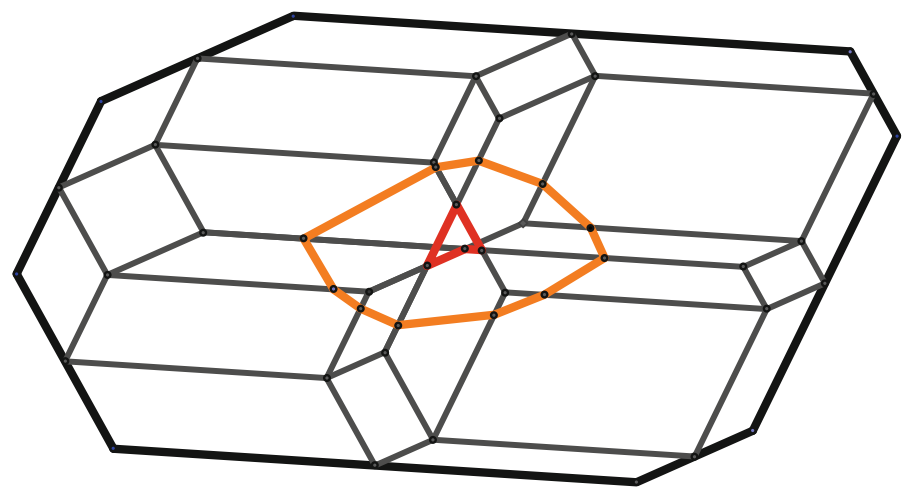

Fig. 9 A rectified area parallel of a parallel opposite sides octogon

Lemma 5.1 If the segment $L(\lambda)$ passes through $B G$, then its support line passes through $E$. If $L(\lambda)$ passes through $G D$, then it is parallel to $P_{i} P_{j+1}$. If $L(\lambda)$ passes through $A B$, then it is parallel to $P_{i+1} P_{j}$.

Proof We may assume that the segment $P_{i} P_{i+1}$ is contained in the $y$-axis and the segment $P_{j} P_{j+1}$ is contained in the $x$-axis. In this case, the area at a point $(x, y)$ is $x y$. Then straightforward calculations prove the lemma.

\subsection{Rectified Area Parallels and Their Cusps}

We shall call the rectified area parallel of level $\lambda$ the union of all segments $L\left(i+\frac{1}{2}\right.$, $j+\frac{1}{2}, \lambda$ ). Thus the rectified area parallels are closed polygons (see Fig. 9 and Applets).

A rectified area parallel has its vertices at the boundaries of the parallelograms $M\left(i-\frac{1}{2}, j-\frac{1}{2}\right)$. Consider a vertex $x$ at the boundary $\left(i-\frac{1}{2}, j\right)$. The rectified area parallel passing through $x$ at the parallelogram $M\left(i-\frac{1}{2}, j+\frac{1}{2}\right)$ will continue the initial polygonal line unless $j=i-1+n$, i.e., $e\left(i-\frac{1}{2}\right)$ is parallel to $e\left(j+\frac{1}{2}\right)$. In the 


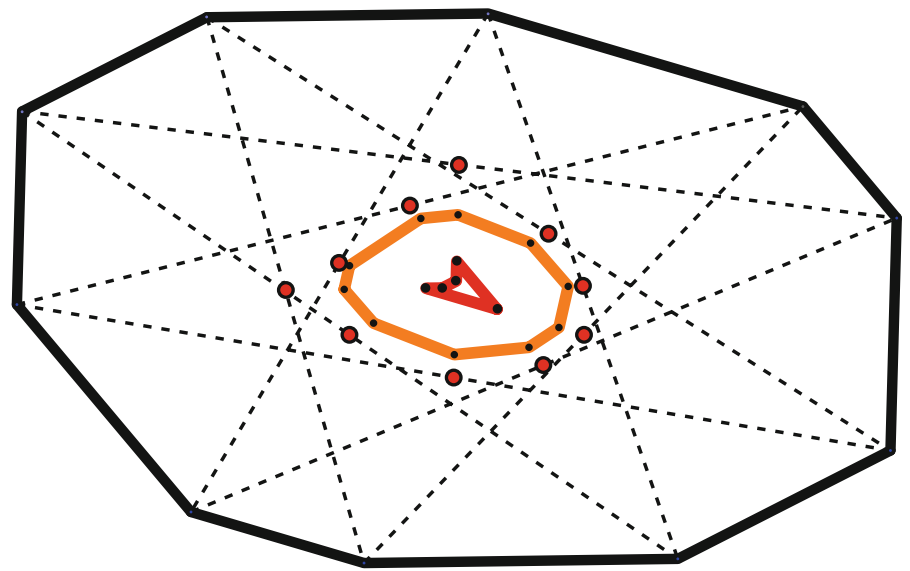

Fig. 10 Area evolute, an equidistant of $\mathcal{Q}$, and the ten midpoints of 1-diagonals (circles). The polygon $\mathcal{P}$ is almost symmetric

latter case, the continuation of the rectified area parallel may occur at the same side of $m\left(i-\frac{1}{2}\right)$ or on the other side. We call $x$ a cusp when the continuation of the area parallel occurs at the same side of $m\left(i-\frac{1}{2}\right)$. We have the following proposition.

Proposition 5.2 A vertex of a rectified area parallel is a cusp if and only if it belongs to the area evolute.

Proof There are two cases to consider: if $x$ is not in the area evolute, then the rectified area parallel will continue in the parallelogram $M\left(i+\frac{1}{2}, i+n-\frac{1}{2}\right)$, and thus $x$ is not a cusp (Fig. 2). If $x$ is in the area evolute, the rectified area parallel will continue in the parallelogram $M\left(i-\frac{1}{2}, i+n+\frac{1}{2}\right)$ (Fig. 3). Close to $x$, the area parallel is contained in the same side of $m\left(i-\frac{1}{2}\right)$, and thus $x$ is a cusp point.

\subsection{Rectified Area Symmetry Set}

The rectified area symmetry set is the locus of self-intersections of the rectified area parallels. It is not easy to describe this set for a general CPOS polygon, so we shall make a simplifying assumption. Denote by $\mathcal{Q}_{\mu}$ the family of equidistants of the paralleldiagonal transform $\mathcal{Q}$ of $\mathcal{P}$. We say that $\mathcal{P}$ is almost symmetric if there exists $\mu_{0}$ such that $\mathcal{Q}_{\mu_{0}}$ contains the area evolute in its interior and all midpoints of 1-diagonals are outside it (Fig. 10).

Under the almost symmetry hypothesis, the polygons $\mathcal{Q}_{\mu}, \mu \geq \mu_{0}$, are rectified area parallels of $\mathcal{P}$. In fact, it follows from Sect. 5.1 that each edge of a rectified area parallel is parallel to the corresponding great diagonal. In particular, using Corollary 4.3 , we have that the rectified area parallel of level $\frac{A(\mathcal{P})}{2}$ is exactly the area evolute of $\mathcal{Q}$.

Proposition 5.3 Under the almost symmetry hypothesis, the rectified area symmetry set of $\mathcal{P}$ coincides with the equidistant symmetry set of $\mathcal{Q}$. 
Proof We first observe that, as a consequence of Proposition 5.2, if a rectified area parallel contains the area evolute in its interior, then it has no self-intersections. Thus, by the almost symmetry hypothesis, the self-intersections of rectified area parallels occur only for those levels corresponding to $\mathcal{Q}_{\mu}, \mu \geq \mu_{0}$. We conclude that the rectified area symmetry set of $\mathcal{P}$ coincides with the equidistant symmetry set of $\mathcal{Q}$.

Acknowledgments The first and second authors wish to thank CNPq for financial support during the preparation of this manuscript.

\section{References}

1. Bobenko, A.I., Suris, Y.B.: Discrete Differential Geometry. Graduate Studies in Mathematics, vol. 98. AMS, Providence, RI (2008)

2. Craizer, M., Teixeira, R.C., da Silva, M.A.H.B.: Area distances of convex plane curves and improper affine spheres. SIAM J. Math. Imaging 1(3), 209-227 (2008)

3. Craizer, M., Teixeira, R.C., da Silva, M.A.H.B.: Affine properties of convex equal-area polygons. Discret. Comput. Geom. 48(3), 580-595 (2012)

4. Giblin, P.J.: Affinely Invariant Symmetry Sets. Geometry and Topology of Caustics, vol. 82. Banach Center Publications, Warsaw (2008)

5. Giblin, P.J., Holtom, P.: The Centre Symmetry Set, vol. 50. Banach Center Publications, Warsaw (1999)

6. Holtom, P.A.: Affine-invariant symmetry sets. Ph.D. Thesis, University of Liverpool (2001)

7. Janeczko, S.: Bifurcations of the center of symmetry. Geom. Dedicata 60, 9-16 (1996)

8. Meyer, M., Reisner, S.: A geometric property of the boundary of symmetric convex bodies and convexity of flotation surfaces. Geom. Dedic. 37, 327-337 (1991)

9. Niethammer, M., Betelu, S., Sapiro, G., Tannenbaum, A., Giblin, P.J.: Area-based medial axis of planar curves. Int. J. Comput. Vis. 60, 203-224 (2004)

10. Domitrz, W., Rios, P.M.: Singularities of equidistants and global symmetry sets of Lagrangian submanifolds. Geom. Dedic. (2013, to appear) 